INPLASY

PROTOCOL

To cite: Hou et al. The effectiveness and safety of moxibustion for treating cancer-related fatigue: a protocol for systematic review and meta-analyses. Inplasy protocol 2021400127. doi: 10.37766/inplasy2021.4.0127

Received: 25 April 2021

Published: 25 April 2021

Corresponding author: xianbing hou

houxianbing007@126.com

Author Affiliation:

Fenghua District Hospital of Traditional Chinese Medicine, Ningbo City, Zhejiang Province

Support: None.

Review Stage at time of this submission: Preliminary searches.

Conflicts of interest: None declared.

\section{The effectiveness and safety of moxibustion for treating cancer- related fatigue: a protocol for systematic review and meta-analyses}

Hou, XB1; Chen, DD2; Cheng, TD3; Wang, D4; Dai, XJ5; Wang, Y6; Cui, BX7; Wang, YY8; Xu, $\mathrm{H}^{9}$; Chen, $\mathrm{HZ}^{10}$.

Review question / Objective: To evaluate the efficacy and safety of various moxibustion methods in the treatment of cancer-related fatigue.

Condition being studied: Studies have also shown that moxibustion has the effects of two-way regulation of body immunity, anti-infection, anti-tumor, analgesic, promotion of metabolism, and delaying aging; in the treatment of malignant tumors, it can inhibit tumor growth and reduce adverse reactions of radiotherapy and chemotherapy. Adjuvant radiotherapy, chemotherapy and surgical treatment to deal with some complications, prolong the survival period of patients and improve the quality of life. Ancient documents and modern research provide a theoretical basis for moxibustion to prevent and treat cancer-related fatigue. Moxibustion is widely used in clinical treatment in China and has achieved good clinical efficacy. This study hopes to further clarify the effectiveness and safety of moxibustion in the treatment of cancer-induced fatigue through systematic reviews and meta-analysis, and to provide references for clinical treatment.

INPLASY registration number: This protocol was registered with the International Platform of Registered Systematic Review and Meta-Analysis Protocols (INPLASY) on 25 April 2021 and was last updated on 25 April 2021 (registration number INPLASY202140127).

\section{INTRODUCTION}

Review question / Objective: To evaluate the efficacy and safety of various moxibustion methods in the treatment of cancer-related fatigue.
Condition being studied: Studies have also shown that moxibustion has the effects of two-way regulation of body immunity, antiinfection, anti-tumor, analgesic, promotion of metabolism, and delaying aging; in the treatment of malignant tumors, it can inhibit tumor growth and reduce adverse 
reactions of radiotherapy and chemotherapy. Adjuvant radiotherapy, chemotherapy and surgical treatment to deal with some complications, prolong the survival period of patients and improve the quality of life. Ancient documents and modern research provide a theoretical basis for moxibustion to prevent and treat cancer-related fatigue. Moxibustion is widely used in clinical treatment in China and has achieved good clinical efficacy. This study hopes to further clarify the effectiveness and safety of moxibustion in the treatment of cancer-induced fatigue through systematic reviews and metaanalysis, and to provide references for clinical treatment.

\section{METHODS}

Participant or population: There are clear and recognized diagnostic criteria and efficacy criteria, and all patients are diagnosed as CRF, regardless of gender, age, and origin of the case.

Intervention: Moxibustion therapy will include all therapies using any type of moxibustion, such as indirect moxibustion, direct moxibustion, heat-sensitive moxibustion, and so on. Mixed therapies based on moxibustion will also be included. Moxibustion therapy, or mixed therapies based on moxibustion will also be include.

\section{Comparator: Conventional treatment.}

\section{Study designs to be included: RCTs.}

Eligibility criteria: There are clear and recognized diagnostic criteria and efficacy criteria, and all patients are diagnosed as CRF, regardless of gender, age, and origin of the case.

Information sources: The two reviewers independently collected data on moxibustion treatments for cancer-related fatigue from CNKI, Wanfang, VIP, Chinese Biomedical Literature Database, Cochrane Library, Pubmed, Embase, CINAHL, Ovidweb, Web of science, from their inception to April 2021. All randomized controlled trials (RCTs) of moxibustion for treating CRF without language restriction were considered for inclusion.

Main outcome(s): Clinical efficacy, including total effective rate or cure rate and fatigue scale score will be accepted as the primary outcomes.

Additional outcome(s): The KPS score, TCM syndrome score, immune indexes (CD3+, CD4+, CD8+, NK), adverse events, will be used as secondary outcomes.

Quality assessment / Risk of bias analysis: This will be conducted using the Cochrane collaboration's risk of bias tool. Two authors will estimate domain risk of bias as follows: sequence generation of randomized, allocation concealment, blinding of participants, personnel and outcome assessment, incomplete outcome data and selective outcome report, and other sources of bias. If there are any disagreements, they will be resolved by the third author.

Strategy of data synthesis: RevMan V.5.3 software will be used in data synthesis. We will express dichotomous data in RR and continuous data in mean difference (MD) or standardized mean difference (SMD). The fixed-effects model will be put into use if $\mathbf{2} 75 \%$, and we will then offer a descriptive analysis or subgroup analysis.

Subgroup analysis: If there are obvious heterogeneity, we will conduct subgroup analysis to identify the sources of heterogeneity. We will conduct subgroup analysis according to different combinations of acupuncture, different course time or different outcome indicators.

Sensitivity analysis: When sufficient trials are available, sensitivity analysis will be performed by sequentially eliding each trial to check the robustness of the final results.

Country(ies) involved: China.

Keywords: Cancer-related fatigue; Moxibustion; Systematic review; Metaanalysis. 
Contributions of each author:

Author 1 - Xianbing Hou.

Email: houxianbing007@126.com

Author 2 - Dandan Chen.

Email: 819328570@qq.com

Author 3 - Tongfei Cheng.

Email: 20964891@qq.com

Author 4 - Dan Wang.

Email: 2537493@qq.com

Author 5 - Xiaojun Dai.

Email: 2398431959@qq.com

Author 6 - Yao Wang.

Email: wangy198302@sohu.com

Author 7 - Bixian Cui.

Email: 459397003@qq.com

Author 8 - Yuanyuan Wang.

Email: wagnyy9208@163.com

Author 9 - Hui Xu.

Email: 598702325@qq.com

Author 10 - Hongzhou Chen.

Email: 2352326376@qq.com 